University of Nebraska - Lincoln

DigitalCommons@University of Nebraska - Lincoln

Papers in Biotechnology

Chemical and Biomolecular Engineering

Research and Publications

6-1-2005

\title{
UV-vis -infrared optical and AFM study of spin-cast chitosan films
}

W H. Nosal

Department of Electrical Engineering, University of Nebraska- Lincoln

D. W. Thompson

University of Nebraska- Lincoln, dthompson2@unl.edu

Sabyasachi Sarkar

Department of Chemical and Biomolecular Engineering, University of Nebrska Lincoln

Anuradha Subramanian

Department of chemical Engineering,University of Nebraska Lincoln., asubramanian2@unl.edu

John A. Woollam

University of Nebraska-Lincoln, jwoollam1@unl.edu

See next page for additional authors

Follow this and additional works at: https://digitalcommons.unl.edu/chemeng_biotechnology

Part of the Biochemical and Biomolecular Engineering Commons

Nosal, W H.; Thompson, D. W.; Sarkar, Sabyasachi; Subramanian, Anuradha; Woollam, John A.; and Yan, L, "UV-vis -infrared optical and AFM study of spin-cast chitosan films" (2005). Papers in Biotechnology. 37. https://digitalcommons.unl.edu/chemeng_biotechnology/37

This Article is brought to you for free and open access by the Chemical and Biomolecular Engineering Research and Publications at DigitalCommons@University of Nebraska - Lincoln. It has been accepted for inclusion in Papers in Biotechnology by an authorized administrator of DigitalCommons@University of Nebraska - Lincoln. 


\section{Authors}

W H. Nosal, D. W. Thompson, Sabyasachi Sarkar, Anuradha Subramanian, John A. Woollam, and L Yan 


\section{Introduction}

Biopolymers, such as cellulose, starch, glycogen and chitin, compose a significant portion of the biomass on the planet. Chitin is a carbohydrate polymer and a major companent in the shells of crustaceans such as shrimp and crabs [1]. Chitin consists of repeated units of $\mathrm{N}$-acetyl-D-glucosamine joined together by $\beta(1 \rightarrow 4)$ linkages. Chitosan is achemically modified form of chitin where the acetyl group attached to the nitrogen atom is replaced by hydrogen, yielding a primary amine group. Both chitin and chitosan have a chemical structure similar in nature to cellulose. The structures of chitin and chitosan are shown in Fig. I.

Work reported in this paper is part of a program to show that chemically modified chitosan coatings on medical implants can be used to provide reduction in immunologicalbased rejection. Control of the clotting reaction (thrombosis) is another potential use of chemically modified chitosan. Chitosan is of interest in this area due to its relative abundance and ease with which it can be chemically modified. The primary amine group found on each chitosan unit provides a site for mild chemical reactions, non-destructive or damaging to the chitosan molecule.

This paper presents the results of vacuum ultraviolet, visible, and infrared wavelength ellipsometry on rpun-cast chitosan films. The purpose is to determine the optical constants of chitosari films prior to chemical modification, over the wide spectral range from the mid-infrared to vacuum ultraviolet regions. In future work, we will report on changes in optical properties due to chemically modified chitosan. The long-range objective is to develop optical methods to monitor surface chemical modifications of biomaterials.

\section{Experimental procedure}

\subsection{Sample preparation}

For this study, thin films of chitosan were produced by spin-casting a solution of chitosan onto $(100)$ orientated silicon wafers (manufactured by Polishing Corporation of 

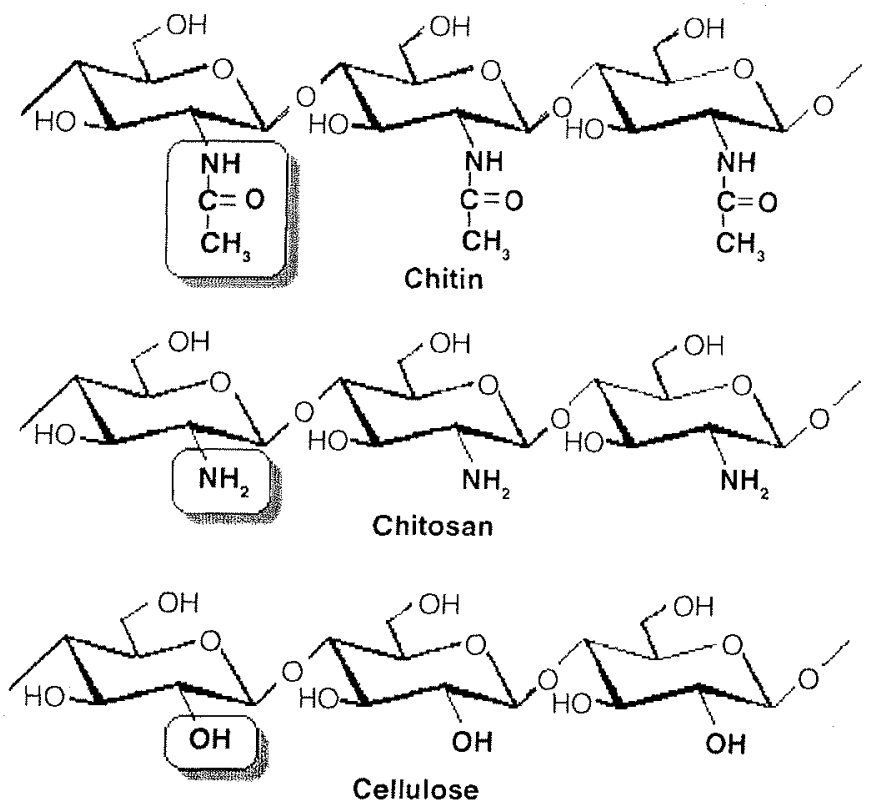

Fig. 1. Structures of chitin and chitosan and their comparison to cellulose.

America). Wafers were 2 in. in diameter, $0.3-0.4 \mathrm{~mm}$ thick, polished on one side and typically had $1.6 \mathrm{~nm}$ of native oxide.

Solutions used to produce films were $2 \%(\mathrm{w} / \mathrm{v})$, signifying that $2 \mathrm{~g}$ of chitosan were dissolved into $100 \mathrm{~mL}$ of solvent. The solvent used was a $2 \%(\mathrm{w} / \mathrm{v})$ aqueous acetic acid solution. Chitosan is soluble in acidic conditions. Powdered chitosan and acetic acid solution were mixed at room temperature on a stir plate. The mixture was stirred for $18 \mathrm{~h}$ yielding a viscous liquid with a faint brown color, with viscosities of around $825 \mathrm{cP}$, as measured on a commercial rotating cylinder viscometer.

The chitosan solutions initially yielded films having numerous small spot and comet defects. It was determined that small undissolved impurity particles were to blame. These particles caused spot defects through capillary action, creating areas of greater film thickness around the particle. Because of the combination of high solution viscosity, small particle size, and possibly a small difference in particle to solution density, an ultracentrifuge was required. By centrifuging the solutions, these particles were precipitated out of the liquid. Centrifugation of solutions for $5 \mathrm{~h}$ with $27,500 \times \mathrm{g}$ acceleration yielded solutions resulting in films virtually free of defects, when using the following spin coating procedure.

Silicon wafers were cleaned by first rinsing in acetone and then in ethanol followed by deionized water. The wafer was air-dried using a pressurized dry air supply, and centered on the P-6000 vacuum chuck and secured through backside vacuum. Five milliliters of chitosan solution were dispensed onto the center of the wafer and allowed to flow to the edges. After systematic tests, $5 \mathrm{~mL}$ was determined to be the optimum amount of chitosan solution. After the solution was allowed to flow over the entire wafer surface, the spin chamber was covered with its lid and the acceleration ramp set at the slowest
Table 1

Sample to sample thickness variations of the spin-casting process for type $\mathrm{A}$ and $\mathrm{C}$ chitosan

\begin{tabular}{lll}
\hline Chitosan & Average thickness $(\mathrm{nm})$ & S.D. $(\mathrm{nm})(n=5)$ \\
\hline Type A & 292.7 & 6.68 \\
Type C & 323.0 & 1.93 \\
\hline
\end{tabular}

setting and the maximum spin speed set to $2500 \mathrm{rpm}$. A Specialty Coating Systems Inc. P-6000 Spin Coater was used. Samples were allowed to spin up to the set speed and spin for $30 \mathrm{~s}$. By pre-loading the solution and using the lowest acceleration ramp, comets and other typical spin coat defects were eliminated or greatly reduced.

After spinning, the sample was removed and allowed to air dry. It was important to neutralize the acidity of the chitosan film. This was done by immersion in $0.25 \mathrm{M} \mathrm{NaOH}$ solution for $15 \mathrm{~min}$ followed by rinsing in deionized water for $15 \mathrm{~min}$. The sample was gently blown dry and placed in a desiccator overnight to ensure as much drying as possible. Average thickness and deviation of the two types of chitosan films is given in Table 1.

\subsection{Sample types}

Chitosan used in this study was supplied by Vanson HaloSource, Seattle, WA. Two grades of chitosan were studied, based on the percentage of chitosan monomer units in the polymer, with the remainder being chitin. The average molecular weight of the chitosan is on the order of 7500 . For the purpose of our studies, grades were designated as types " $\mathrm{A}$ " and "C", having $82.1 \%$ and $93.8 \%$ chitosan polymer units, respectively. The chitosan percentage is also referred to as "de-acetylation factor", since the chemical process of making chitosan involves reacting chitin to remove the acetyl functional group.

For this work, five samples of each of the two grades of chitosan were spun-cast using the aforementioned technique.

\subsection{UV-vis spectroscopic ellipsometry}

Spectroscopic ellipsometry (SE) is a non-destructive method used to determine film thickness and optical constants $[2-4]$ : The change in the polarization state of a reflected light beam is measured over a wide range of wavelengths and at one or more angles of incidence, called variable angle spectroscopic ellipsometry (VASE). The sample complex reflection coefficients for polarized light beams (parallel and perpendicular to the plane of incidence), are given by the Fresnel coefficients $R_{\mathrm{p}}$ and $R_{\mathrm{s}}$, respectively. Ellipsometric parameters $\psi$ and $\Delta$ are related to these coefficients by:

$\rho \equiv \tan (\psi) \mathrm{e}^{i \Delta}=\frac{R_{\mathrm{p}}}{R_{\mathrm{s}}}$

The weighted mean-square difference between calculated and measured values is calculated, and then minimized using the Levenberg-Marquardt algorithm by varying the model 
parameters. The thickness and optical constants are obtained as a result of regression fits.

Data in the vacuum ultraviolet through near infrared were taken on a commercial variable angle spectroscopic ellipsometer (VUV-VASE). Chitosan is hydroscopic and will absorb water resulting in swelling. Early attempts to acquire and optically model the ellipsometric data to obtain thickness using a visible spectroscopic ellipsometer were unacceptable because chitosan water absorption from air expanded the thickness. Therefore, an ultraviolet ellipsometer using a dry nitrogen exchange gas filled chamber was used to acquire ellipsometric data from 0.75 to $9.0 \mathrm{eV}$ (approximately $130-1700 \mathrm{~nm}$ ). This prevented absorption of water vapor by the films. Using the 500-1700 nm data range and a Cauchy dispersion model for the SE data, resulted in meaningful evaluation of thickness.

\section{Data analysis}

\subsection{Visible spectroscopic ellipsometry}

It is also important to note the two main forms of optical constants and how they are related $[2,3]$. These are $n$ and $k$ as well as $\varepsilon_{1}$ and $\varepsilon_{2}$. These are related through the equations:

$\varepsilon_{1}=\frac{n^{2}-k^{2}}{\mu}$

$\varepsilon_{2}=\frac{2 n k}{\mu}$,

where $\mu$ is the magnetic permeability of the material, which for chitin and chitosan has a value of 1 . The analysis software used is able to convert between $n$ and $k$ to $\varepsilon_{1}$ and $\varepsilon_{2}$ automatically and plots of $n, k$, and $\varepsilon_{1}, \varepsilon_{2}$ are used throughout this paper. The $\varepsilon_{1}$ and $\varepsilon_{2}$ format are most appropriate when discussing the physics. The $n$ and $k$ format are used extensively in engineering applications.

It is of importance to also note that $k$ is related to the penetration depth $\alpha$ in the following equation:

$k=\frac{\alpha \lambda}{2 \pi}$,

where $\alpha$ is the penetration depth and $\lambda$ is the wavelength of the incident light.

Visible SE data from chitosan films were optically modeled assuming a Cauchy dispersion model, to represent the index of refraction, $n$, as a slowly varying function of wavelength. A Cauchy model assumes no absorption so the extinction coefficient, $k$, is assumed to be zero. The Cauchy equation is:

$n(\lambda)=A+\frac{B}{\lambda^{2}}+\frac{C}{\lambda^{4}}$,

where the three parameters in the dispersion model are $A, B$, $C$, and $\lambda$ is the wavelength. For the chitosan samples in this study, $A, B, C$, and thickness were fit by regression analysis from experimental $S E$ data.

For the no absorption assumption to be valid, the model must fit over a limited wavelength range. Even though data were taken from 300 to $1700 \mathrm{~nm}$, the Cauchy model was fit using data from 500 to $1700 \mathrm{~nm}$, in which there was clearly no absorption. This excludes the UV wavelengths in which there was pronounced adsorption of light, thus violating the assumption of the Cauchy model.

Absorption in the ultraviolet was accounted for by including two Gaussian line-shape oscillators, then allowing the position (in photon energy), amplitude and width of each oscillator to float in the regression analysis.

\subsection{Infrared spectroscopic ellipsometry}

Infrared data were taken on a commercial spectroscopic ellipsometer $[3,4]$. This was modified to include a custommade sample holder providing dry air purge gas around the chitosan sample. This prevented water absorption by the chitosan films during data acquisition.

To successfully model chitosan films in the infrared, data were taken on each blank silicon wafer before chitosan was spin-cast. Substrate characterization was required in the infrared because a small difference in dopant concentration of each wafer had a significant effect on the free carrier concentration and thus on optical properties.

SE data on each wafer were modeled to a Drude model to account for absorption in the infrared due to free carriers $[5,6]$. The Drude model is obtained from the Lorentz oscillator model by setting the restoring force to zero. The Lorentz oscillator model for the dielectric function is given as:

$\hat{\varepsilon}(E)=\varepsilon_{1 \infty}+\sum_{n} \frac{A_{n}}{E_{c n}^{2}-E^{2}-i B_{n} E}$,

where $\varepsilon_{1 \infty}$ is the real part of the dielectric function as the energy goes to infinity, and $A_{n}, B_{n}$ and $E_{\mathrm{c} n}$ are the strength, broadening (loss), and center energy of the $n$th oscillator, respectively. Setting the centering energy to zero, as well as writing $A$ and $B$ in terms of $\rho$, the resistivity of the material and $\tau$, the mean scattering time between collisions of the free carriers, gives the " $\rho-\tau$ " form of the Drude model [2]:

$\hat{\varepsilon}(E)=\varepsilon_{1 \infty}-\frac{\hbar^{2} / \varepsilon_{0}}{\rho \tau\left(E^{2}+(i \hbar E / \tau)\right)}$.

This relates the resistivity and mean scattering time to the dielectric function and allows any differences in free carrier density from sample to sample to be accounted for [6]. The layer thickness of each chitosan spin-cast sample was determined from SE data taken in a dry nitrogen purged ellipsometer, and using a Cauchy model fit, as described earlier.

Infrared SE data were modeled using harmonic oscillators to account for absorptions due to chemical bond vibrations [7-16]. Oscillator center energy, amplitude, and broadening were fit, one parameter at a time, for each individual chemical bond related peak in the data. Once this initial fit 
scheme was completed, the energy, amplitude, and broadening were simultaneously fit for each individual oscillator. Upon completion of fitting for each oscillator individually, data were fit for in groups of two or three oscillators, and finally all three fit parameters were floated for all oscillators simultaneously in a total parameter fit. This step-wise fitting procedure was required to arrive at a best fit, while preventing some oscillators from showing unphysical results such as very small (or large) broadening, or negative amplitudes. Beginning a fit by allowing too many fit parameters to float at once resulted in a high probability that the fit would go awry.

\subsection{Atomic force microscopy (AFM)}

For a better understanding of surface features and roughness, atomic force microscopy was used to study spin-cast chitosan films in air. AFM topographic images were obtained at room temperature using a multimode AFM with a Nanoscope III controller (Digital Instruments Veeco Metrology Group, Santa Barbra, CA). Several scan sizes were used to examine surface features of spin-cast chitosan. Four sizes were used, a $300 \mathrm{~nm}$ square, a $1 \mu \mathrm{m}$ square, a $2 \mu \mathrm{m}$ square, and a $5 \mu \mathrm{m}$ square. A Digital Instruments multimode atomic force microscope (AFM) in tapping mode was used to produce topography images of the spin-cast chitosan films in an air ambient state. Both type $\mathrm{A}$ and $\mathrm{C}$ chitosan were examined. Results are presented in Section 4.3.

\section{Results}

\subsection{VUV-vis-near IR optical properties of chitosan}

VUV-vis-near IR data fits show that the thickness of the chitosan spin-casting is repeatable to within a few percent from sample to sample.

When the model allowed uniaxial anisotropy, the optical constants lying in the plane were distinguishable from those oriented out of the plane. Variable angle spectroscopic ellipsometry is very sensitive to differences in index of refraction. Fig. 2 shows the index of refraction in the non-absorbing region for only type A spin-cast chitosan films, demonstrating the sensitivity of ellipsometry to optical anisotropy. Type C is virtually identical over this wavelength range and is not shown here. Optical anisotropy of spin-cast polymers is strain induced, a result of sample spinning. The wide spectral range SE data of the in-plane versus out-of-plane index of refraction are sensitive to index anisotropy as small as 0.001 , which is less than $0.1 \%$ of the index for chitosan.

Most polymers and biopolymers absorb in the UV, including chitosan. The result is a non-zero extinction coefficient for wavelengths shorter than approximately $225 \mathrm{~nm}$. Fig. 3 shows $n$ and $k$ for type A chitosan as a function of wavelength from 138 to $300 \mathrm{~nm}$. Fig. 4 shows the same results, but for type $\mathrm{C}$ chitosan.

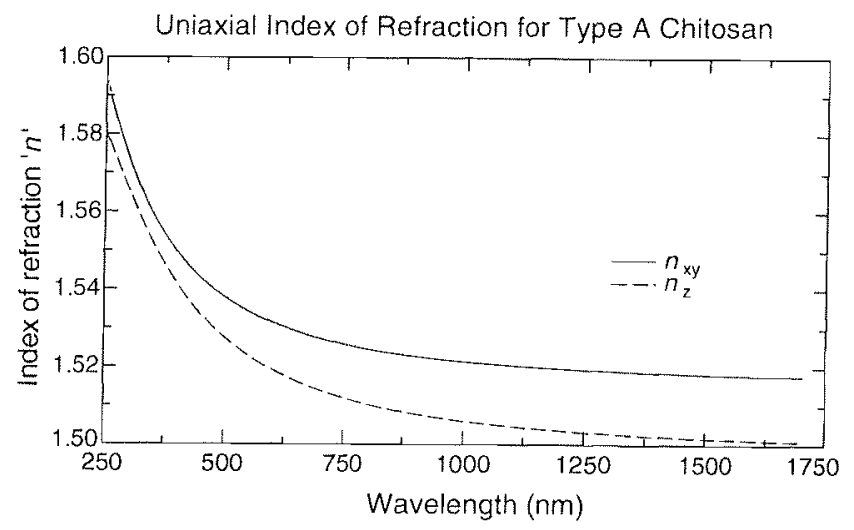

Fig. 2. Plot of anisotropy in index of refraction for type A chitosan.

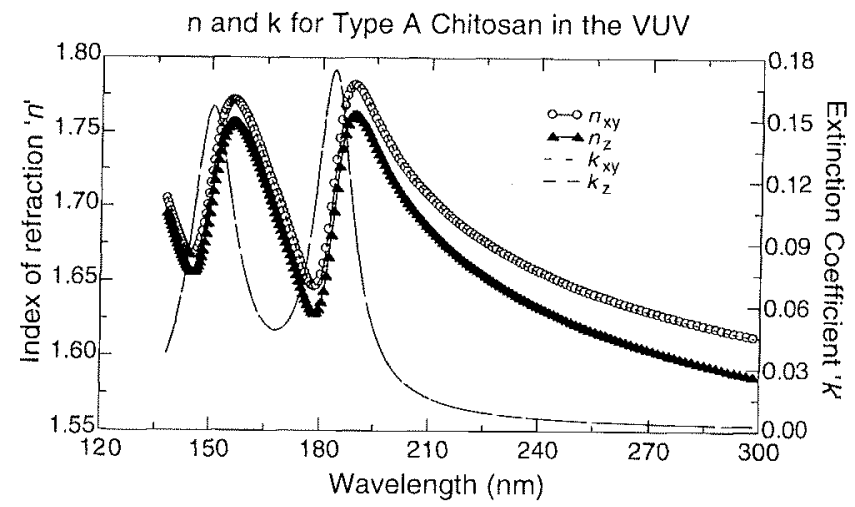

Fig. 3. Optical constants for type A chitosan as a function of wavelength.

The following can be stated from Figs. 3 and 4. First, the onset of absorption occurs at a slightly shorter wavelength for type C chitosan than for type A, $190 \mathrm{~nm}$ versus $200 \mathrm{~nm}$. Second, the initial absorption peak for each type is centered at a different wavelength, $185 \mathrm{~nm}$ for type $A$ and $172 \mathrm{~nm}$ for type $\mathrm{C}$. The chemical differences in type $\mathrm{A}$ and $\mathrm{C}$ chitosan are the likely reason for this. Third, it is important to note that there are clearly two oscillators present in the data, but a third not fully seen. It is also important to note that the second absorption peak at $149 \mathrm{~nm}$ is noticeably smaller for

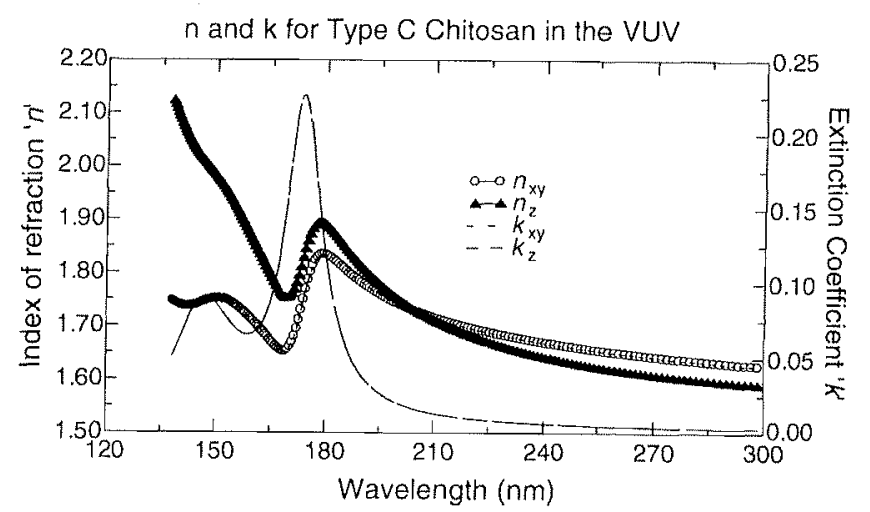

Fig. 4. Optical constants for type $C$ chitosan as a function of wavelength. 
the type $\mathrm{C}$ chitosan than for the type $\mathrm{A}$. This peak is due to the $\pi$-bond absorption of the carbonyl group contained in the chitin units of the polymer. The ratio of the area under this peak should correspond to the volume ratio of the occurrence of the carbonyl group between types A and C. The ratio as stated by the manufacturer is 2.89 , and the ratio of the areas under the peaks in $\varepsilon_{2}$ is approximately 2.2 . These ratios agree to within $25 \%$ of each other. The areas under the peaks centered at $6.3 \mathrm{eV}$ are within a few percent of each other between type $\mathrm{A}$ and $\mathrm{C}$ samples. The fact that the $8.3 \mathrm{eV}$ peak is smaller in area for the sample with a smaller occurrence of the carbonyl group adds confirmation to the statement that the $\pi$-bond electrons in this functional group are what lead to this particular absorption.

\subsection{Infrared optical properties of chitosan}

The optical constants of spin-cast chitosan films were determined in the IR. Data were collected over the spectral range from 230 to $7900 \mathrm{~cm}^{-1}$, corresponding to wavelengths from approximately 1.27 to $43.2 \mu \mathrm{m}$. Both the index of refraction and extinction coefficient ( $n$ and $k$ ), as well as the real and imaginary parts of the dielectric function $\left(\varepsilon_{1}\right.$ and $\left.\varepsilon_{2}\right)$ were determined from regression-based data analysis software. Typically, these fits resulted in mean square errors of between 20 and 25 . In the case of chitosan films, the optical constants were determined over a range of $750-4000 \mathrm{~cm}^{-1}$, as this is the typical spectral range containing chemical bond vibration energies. A plot showing the index of refraction for type A chitosan is given in Fig. 5. These resulted from oscillator-based analysis of the raw $\psi$ and $\Delta$ data using eight different oscillators. This figure shows the in-plane ( $x y$-plane) and out-of-plane (z-axis) components of index. Figs. 6 and 7 show the corresponding infrared extinction coefficients for types $\mathrm{A}$ and $\mathrm{C}$, respectively.

Table 2 lists the chemistries detected using IR-VASE. In the chemical identification, two main points must be mentioned. First, the broad peak centered at around $3320 \mathrm{~cm}^{-1}$ is the result of $\mathrm{O}-\mathrm{H}$ stretch $[4,8]$. The raw data in this region

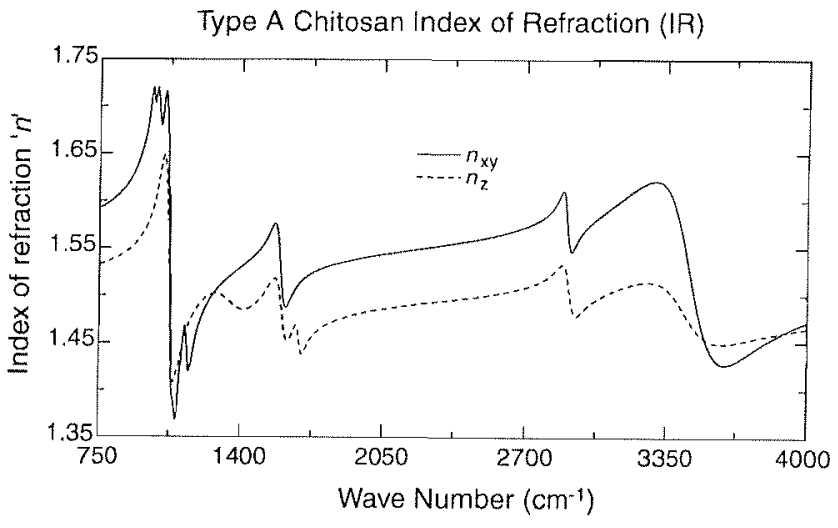

Fig. 5. In-plane $(x y)$, and out-of-plane (z) index of refraction for type A chitosin in the infrared.

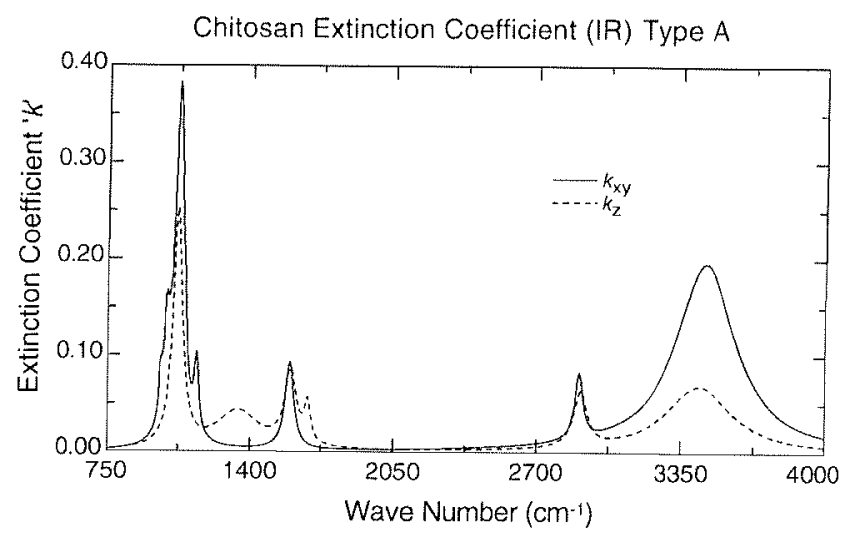

Fig. 6. Extinction coefficient $(k)$ as a function of number for type A chitosun in the infrared.

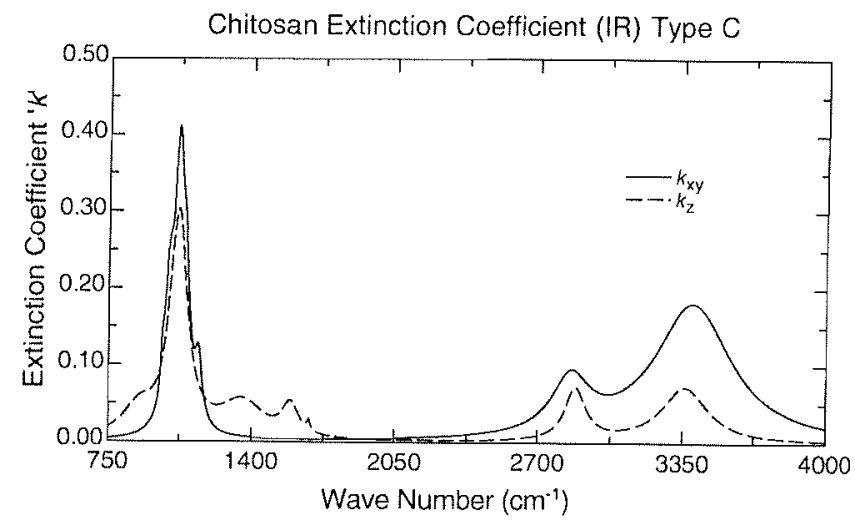

Fig. 7. Extinction coefficient $(k)$ as a function of wavelength for type $\mathrm{C}$ chitosan in the infrared.

show a slight bimodal shape in the peak in $\psi$. However, attempts to model this using two independent peaks failed to find a unique solution in the regression, as the degree of overlap between the two oscillator peaks was too large and led to high parameter correlation. Nevertheless, the $3320 \mathrm{~cm}^{-1}$ peak is actually composed of two overlapping oscillator absorptions.

The shift of the $\mathrm{O}-\mathrm{H}$ peak to such a low wavenumber was the result of strong hydrogen bonding of the $\mathrm{OH}$ groups [1]. Strong hydrogen bonding is common in solid chitin/chitosan polymer when the polymer is in a crystalline form where the

Table 2

Functional group peak assignments for solid state chitosan

\begin{tabular}{ll}
\hline Position $\left(\mathrm{cm}^{-1}\right)$ & Functional group \\
\hline 3320 & $\mathrm{OH}$ stretch \\
2900 & $\mathrm{NH}$ stretch \\
1660 & $\mathrm{C}-\mathrm{N}-\mathrm{H}$ bending \\
1600 & $\mathrm{C}=\mathrm{O}$ \\
1160 & $\mathrm{C}-\mathrm{O}$ \\
1050 & $\mathrm{C}-\mathrm{C}$ fingerprint \\
1030 & $\mathrm{C}-\mathrm{N}$ \\
970 & $\mathrm{C}-\mathrm{C}, \mathrm{C}-\mathrm{N}$ fingerprint \\
\hline
\end{tabular}


Type A and C Extinction Coefficients (IR)

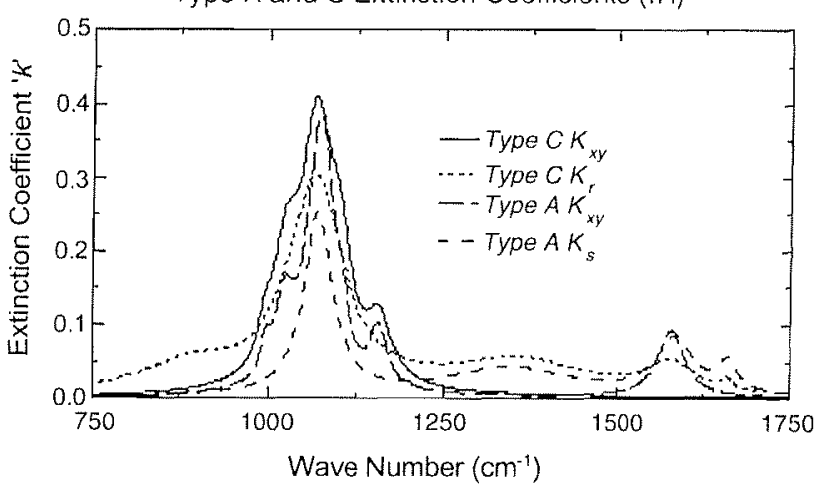

Fig. 8. Fingerprint and carbonyl peak regions showing both in-plane and out-of-plane extinction coefficient for type $\mathrm{A}$ and $\mathrm{C}$ chitosan.

polymer chains are packed side by side [I]. The lack of a peak in the $3500-3700 \mathrm{~cm}^{-1}$ range signified that indeed the chitosan films made for this study had an ordered structural form to some extent, and their polymer chains are likely packed side by side to a large extent.

Secondly, note that $950-1100 \mathrm{~cm}^{-1}$ is an area of overlapping peaks. This energy range corresponds to the "fingerprint region" of the molecule $[5,8]$, with absorptions due to a variety of $\mathrm{C}-\mathrm{C}, \mathrm{C}-\mathrm{O}$, and $\mathrm{C}-\mathrm{N}$ single bond vibrations.

Infrared spectroscopic ellipsometry (IR-SE) is sensitive to molecular orientation anisotropy in the sample. This sensitivity can be seen in Fig. 8 as the differences in values for the optical constants for each chemical peak from the in-plane (xy-plane) and the out of sample plane (z-axis).

Of special note is the occurrence of a peak at $1150 \mathrm{~cm}^{-1}$ for the in-plane $\varepsilon_{2}$ for both type $\mathrm{A}$ and $\mathrm{C}$ chitosan and its absence in the out-of-plane, or $z$-direction. This peak corresponds to the $\mathrm{C}-\mathrm{O}-\mathrm{C}$ groups contained within the backbone of the polysaccharide chain [1,5]. Detection of this functional group within the plane of the sample and not out of the plane leads to the conclusion that the polymer chains lie predominately within the plane. This is physically reasonable, in that spin-casting of polymer films forces the polymer chains to lay in-plane, due to their long dimensional chains. Fig. 8 shows a more detailed plot of $k$ for this region for both type $\mathrm{A}$ and $\mathrm{C}$ chitosan showing in-plane and out-of-plane contributions. It is noted that the peaks in the area of $1150-1165 \mathrm{~cm}^{-1}$ occur only for the in-plane data and not for the out-of-plane data. This leads to the conclusion that these bonds lie within the plane of the sample.

IR-SE can also quantitatively distinguish between the two types of chitosan (A and C) studied in this work. This is possible because IR-SE, unlike absorption/transmission IR spectroscopy, can determine absolute optical constants. The area under the peaks of the absolute $\varepsilon_{2}$ is proportional to the occurrence of particular chemical bonds in the sample. Fig. 9 shows a plot of $\varepsilon_{2}$ as a function of wavenumber including the $\mathrm{C}=\mathrm{O}$ peak at $1600 \mathrm{~cm}^{-1}$. The areas under the peaks in $\varepsilon_{2}$ correspond to the relative occurrence of the carbonyl group in the samples. Since the carbonyl peak only occurs in unreacted
Type A vs. C Out of Plane Imaginary Dielectric Constant

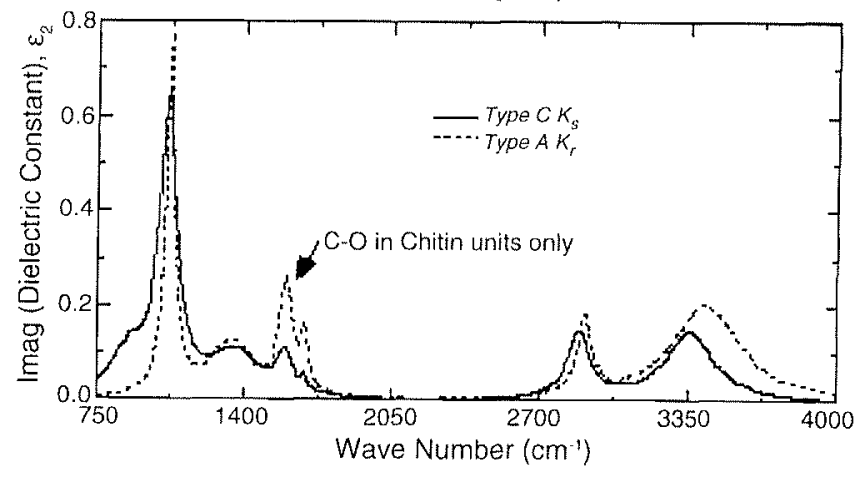

Fig. 9. Out-of-plane component of $\varepsilon_{2}$ with the carbonyl peak identified.

chitin units, the ratio of the type $A$ to $C$ areas correspond to the ratio of chitin units between these two samples. The manufacturer's specified ratio of carbonyl occurrence for type A to $C$ chitosan is 2.89 . The ratio of the areas under the peaks in $\varepsilon_{2}$, as obtained from the modeling software used is 2.54 . These ratios are within $20 \%$ of the ratio given by the chitosan supplier, which may have error limits of its own.

\subsection{Results of AFM studies on spin-cast chitosan films}

Tapping mode AFM images yielded information about the surface features of the spin-cast chitosan films. Fig. 10 shows a tapping mode AFM images of type A chitosan spin-cast film.

For the purpose of this paper, larger scan sizes of $1 \mu \mathrm{m} \times 1 \mu \mathrm{m}, 2 \mu \mathrm{m} \times 2 \mu \mathrm{m}$, and $5 \mu \mathrm{m} \times 5 \mu \mathrm{m}$ are not shown, however, their information was used in obtaining RMS roughness values. AFM images of type C chitosan, seen in Fig. 11, show that type $C$ films have larger feature sizes than type A. Table 3 gives RMS roughness values for

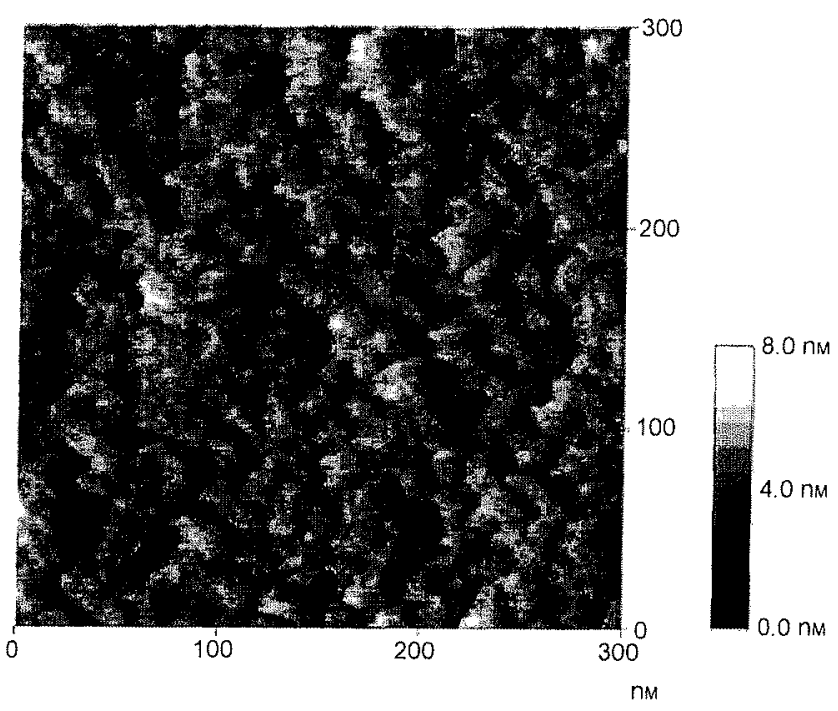

Fig. 10. Tapping mode AFM image of type A chitosan measuring $300 \mathrm{~nm} \times 300 \mathrm{~nm}$. 


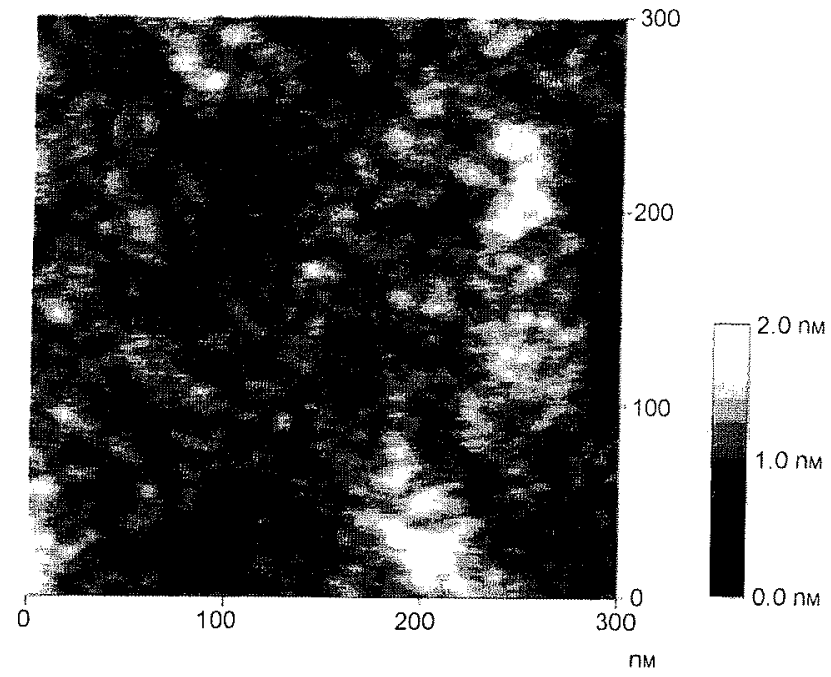

Fig. 11. Tapping mode AFM image of type $C$ chitosan measuring $300 \mathrm{~nm} \times 300 \mathrm{~nm}$.

Table 3

AFM-derived RMS roughness of spin-cast chitosan films

\begin{tabular}{ll}
\hline Type A scan size & RMS roughness $(\mathrm{nm})$ \\
\hline $300 \mathrm{~nm} \times 300 \mathrm{~nm}$ & 0.9 \\
$1 \mu \mathrm{m} \times 1 \mu \mathrm{m}$ & 0.9 \\
$2 \mu \mathrm{m} \times 2 \mu \mathrm{m}$ & 1.0 \\
S.D. & 0.065 \\
Type C scan size & RMS roughness $(\mathrm{nm})$ \\
\hline $300 \mathrm{~nm} \times 300 \mathrm{~nm}$ & 0.3 \\
$1 \mu \mathrm{m} \times 1 \mu \mathrm{m}$ & 0.3 \\
$2 \mu \mathrm{m} \times 2 \mu \mathrm{m}$ & 0.3 \\
S.D. & 0.023 \\
\hline
\end{tabular}

both types of chitosan. This shows that the roughness of type $\mathrm{C}$ samples is likely less than type A.

The nanometer scale morphology also appears different for each image scale when comparing type A chitosan to type $\mathrm{C}$ chitosan. For example, at the $300 \mathrm{~nm}$ scale, the type $\mathrm{C}$ is smoother but shows size non-uniformity for the in-plane features. These features are also larger on average for the type $\mathrm{C}$ chitosan than the type A chitosan. The surface morphology at this scale could be the result of small regions of amorphous polymer contained within a polycrystalline-like matrix or vice versa.

These results show, for both types of chitosan, that spincasting can yield films with RMS surface roughness of approximately $0.9 \mathrm{~nm}$ for type $A$ and on the order of $0.3 \mathrm{~nm}$ for type $\mathrm{C}$ chitosan.

\section{Conclusions}

Spin-cast chitosan films comprised of two types of chitosan have been studied using AFM, and spectroscopic ellipsometry. Optical constants for both types of chitosan have been determined for a wide spectral range. Anisotropy is detectible in all spectral ranges from VUV to infrared. Index of refraction has been shown for spin-cast samples over the near infrared and visible wavelengths, as was shown in Fig. 2. Optical constant determination in the infrared has allowed for chemical identification of chitosan films and identification of chemical anisotropy.

AFM studies show that smooth films can be made by spincasting, with RMS roughnesses of approximately $0.9 \mathrm{~nm}$ for type A chitosan and $0.3 \mathrm{~nm}$ of RMS roughness for type C.

\section{Acknowledgements}

Special thanks are given to Corey Bungay of J.A. Woollam Company for acquiring the VUV-VASE data used in this work, Tom Tiwald for editorial comments, the U.S. Army Contract number W911NF-04-2-0011, and the College of Engineering and Technology at the University of Nebraska at Lincoln for financial support.

\section{References}

[1] D. Alain, D. Monique, Chitosan: structure-properties relationship and biomedical applications, in: S. Dumiprin (Ed.), Polymeric Biomaterials, second ed., Marcel Dekker, New York, 2002, pp. 187-212 (Chapter 9).

[2] H.G. Tompkins, W.A. McGahan, Spectroscopic Ellipsometry and Reflectometry: A User's Guide, Wiley, New York, 1999.

[3] J.A. Woollam, B. Johs, C.M. Herzinger, J. Hilfiker, R. Synowicki, C.L. Bungay, Overview of variable angle spectroscopic ellipsometry (VASE). Part I. Basic theory and typical application, Crit. Rev. Opt. Sci. Technol. CR72 (1999) 3-28.

[4] B. Johs, J.A. Woollam, C.M. Herzinger, J. Hilfiker, R. Synowicki, C.L. Bungay, Overview of variable angle spectroscopic ellipsometry (VASE). Part II. Advanced applications, Crit. Rev. Opt. Sci. Technol, CR72 (1999) 29-58.

[5] T. Tiwald, D. Thompson, J.A. Woollam, W. Paulson, R. Hance, Ap. plication of variable angle spectroscopic ellipsometry to the determination of free carrier concentration depth protiles, Thin Solid Films 313-314 (1998) 661-666.

16] F. Wooten, Optical Properties of Solids, Academic Press, San Diego, 1972.

17] D. Naumann, FT-infrared and FT-Raman spectroscopy in biomedical research, in: H.U. Gremlich, B. Yan (Eds.), Infrared and Raman Spectroscopy of Biological Materials, Marcel Dekker, New York, 2001. pp. 323-377.

[8] B. Stuart, Biological Applications of Infrared Spectroscopy, Wiley, Chichester, UK, 1997.

[9] D.G. Castner, B.D. Ratner, Biomedical surface science: foundations to frontiers, Surf. Sci. 500 (2002) 28-60.

[10] M. Diem, Introduction to Modern Vibrational Spectroscopy, Wiley, New York, 1993.

[11] R.H. Garrett, C.M. Grisham, Biochemistry, second ed., Harcourt College Publishers, Fort Worth, TX, 1999.

[12] B. Kasemo, Biological surface science, Surf. Sci. 500 (2002) $656-677$

[13] M. Mantsch, D. Chapman, Infrared Spectroscopy of Biomaterials, Wiley, New York, 1996

[14] J.L. Brash, P.W. Wojciechowski, Interfacial Phenomenon and Bioproducts, Marcel Dekker, New York, 1996.

[15] M. Tirrell, E. Kokkoli, M. Biesalski, The role of surface science in bioengineered materials, Surf. Sci. 500 (2002) $61-83$.

[16] C.J. Hirschmugl, Frontiers in infrared spectroscopy at surfaces and interfaces, Surf. Sci. 500 (2002) 577-604. 